\title{
Die erste Kaiserin
}

Ein Mythos zum ,geistigen Zusammenhalt' der Frauenbewegung

Le mythe de la première impératrice, ou comment perpétuer l'héritage féministe sous le III Reich

The First Empress - a Myth on the 'Spiritual Cohesion' of the Women's Movement

\section{Christina Stange-Fayos}

\section{(2) OpenEdition}

Journals

Édition électronique

URL : http://journals.openedition.org/ceg/5060

DOI : $10.4000 /$ ceg.5060

ISSN : 2605-8359

Éditeur

Presses Universitaires de Provence

Édition imprimée

Date de publication : 2 mai 2019

Pagination : 251-264

ISBN : 979-10-320-0214-8

ISSN : 0751-4239

Référence électronique

Christina Stange-Fayos, „Die erste Kaiserin“, Cahiers d'Études Germaniques [Online], 76 | 2019, Online erschienen am: 02 November 2020, abgerufen am 25 Januar 2021. URL: http:// journals.openedition.org/ceg/5060 ; DOI: https://doi.org/10.4000/ceg.5060 


\section{Die erste Kaiserin \\ Ein Mythos zum , geistigen Zusammenhalt‘
der Frauenbewegung}

Christina STANGE-FAYOS

Université Toulouse-Jean Jaurès, CREG (EA 4151)

Der Gleichschaltung durch die nationalsozialistischen Machthaber beugte der Bund Deutscher Frauen (BDF) vor, indem er sich am 15. Mai 1933 selbst auflöste. Dies bedeutete das Ende der ersten deutschen Frauenbewegung, die sich seit den 1870er Jahren reichsweit organisiert hatte und auf große Erfolge verweisen konnte - nicht zuletzt auf das 1918 erlangte Frauenwahlrecht.

Dieses Wahlrecht war aktiv und passiv, so dass viele Persönlichkeiten des BDF in der Weimarer Republik zu Reichstagsabgeordneten avancierten. So z.B. die langjährige Vorsitzende des Bundes, Gertrud Bäumer (1873 - 1954), die 1919 in die verfassunggebende Versammlung, dann in den Reichstag gewählt wurde (DDP). 1920 wurde Gertrud Bäumer als Ministerialrätin in das Reichsinnenministerium berufen, daneben war sie von 1926 bis 1933 Delegierte der Reichsregierung beim Völkerbund in Genf.

Der Nationalsozialismus hatte nie einen Hehl aus seiner Abneigung gegenüber Frauen in der Politik gemacht. So konnten sie zwar Mitglied der NSDAP werden, jedoch keine parteiinternen Funktionen einnehmen. Frauen sollten auf den ihnen „wesensgemäßen“ Ort ihres Wirkens beschränkt werden: die Familie und das Haus. ${ }^{1}$ Alfred Rosenberg forderte die Emanzipation der Frau von der Frauenemanzipation. Im März 1933 wurde Gertrud Bäumer von ihrem Posten als Ministerialrätin im Reichsinnenministerium zuerst beurlaubt und schließlich wurde ihr nach $\S 4$ des Gesetzes zur Wiederherstellung des Berufsbeamtentums vom 7. April 1933 wegen „,politischer Unzuverlässigkeit“ gekündigt. Das Jahr 1933 bedeutete infolgedessen für Gertrud Bäumer einen Schnittpunkt: Aus dem Beruf gedrängt und aller öffentlichen Ämter enthoben, nutzte sie die ihr nun zur Verfügung stehende Zeit - weg von Berlin - für historische Studien, Reisen und schriftstellerische Arbeiten. Sie rechnete sich selbst seit ihrer Entlassung der inneren Emigration zu, die sie im weitesten Sinne dem Widerstand zuordnete. ${ }^{2} 1936$ veröffentlichte sie ihr aus dieser Zeit bekanntestes

1. Renate Wiggershaus, Frauen unterm Nationalsozialismus, Wuppertal, P. Hammer, 1984, S. 15.

2. Ralf Schnell, Literarische Innere Emigration 1933-1945, Stuttgart, Metzler, 1976; siehe auch Schnell, Dichtung in finsteren Zeiten. Deutsche Literatur und Faschismus, Hamburg, Rororo, 1998, insb. S. 120-160. 
und meistverkauftes Werk, Adelheid, Mutter der Königreiche ${ }^{3}$. Adelheid von Burgund war, als Gemahlin Lothars von Italien, von 947 bis 950 Königin von Italien. Dann, als Gemahlin Ottos des Großen, von 951 bis 973 ostfränkische Königin und wieder Königin von Italien sowie von 962 bis 973 Kaiserin des römischen Reiches. Sie wurde 1097 heiliggesprochen. ${ }^{4}$ Bis 1945 erschienen 6 Auflagen der Adelheid Bäumers. 1955 erreichte die Auflage eine Höhe von 100000 Exemplaren. Wie sehr das Buch bei der deutschen Leserschaft ankam, lässt sich übrigens nicht nur aus den hohen Auflagen, sondern auch daraus ersehen, dass die Adelheid 1941 als Vorlage für ein Opernlibretto diente... in der Forschung ist es bislang kaum rezipiert worden. Im Folgenden soll daher den Fragen nachgegangen werden: wie und warum rekonstruiert Gertrud Bäumer den Adelheidmythos? Was kann die begeisterte Rezeption dieses Werkes erklären?

$* * *$

Als sich der BDF am 15. Mai 1933 auflöste, reagierte Gertrud Bäumer verhältnismäßig gelassen. Der Zerfall der Vereinsstrukturen konnte nämlich, ihrer Meinung nach, das Wesen der Frauenbewegung nicht zerstören. Es ging nun darum, die emanzipatorischen Werte, also ein immaterielles Erbe, weiterzutragen und $\mathrm{zu}-$ geben. 1873 in Westfalen geboren, 1954 in Bielefeld gestorben, kann Gertrud Bäumer als typische Vertreterin der so genannten alten (oder „ersten“) Frauenbewegung gelten, und zwar durch folgende Charakteristika: sie war bildungsbürgerlicher Herkunft, protestantisch, ledig, und aus wirtschaftlichen Gründen gezwungen, den Beruf der Lehrerin zu ergreifen. 1898 zog sie nach Berlin. Ab 1899 teilte sie mit der 25 Jahre älteren Helene Lange (geboren 1848, gestorben 1930) eine produktive Arbeits- und Lebensgemeinschaft, engagierte sich zunehmend in der Frauenbewegung und entwickelte eine rege Publikationstätigkeit. 1904 promovierte sie an der philosophischen Fakultät der Friedrich-Wilhelms-Universität Berlin zur Entstehungsgeschichte von Goethes Satyros. Von 1910-1919 war sie Vorsitzende des BDF, den sie auch nach 1919 als stellvertretende Vorsitzende bis zu dessen Auflösung entscheidend prägte. Sie war während des Ersten Weltkriegs Mitbegründerin des „Nationalen Frauendienstes“, der versuchte, alle zur Verfügung stehenden Frauen für die Kriegsfürsorge zu gewinnen. ${ }^{5}$ Was ihr publizistisches Schaffen betrifft, muss man hinzufügen, dass sie von 1907-1912 die Schriftleitung des Periodikums Neue Bahnen innehatte. Seit 1912 arbeitete sie in der Redaktion der von Friedrich Naumann herausgegebenen Zeitschrift Die Hilfe mit. ${ }^{6}$ Somit erscheint sie in der

3. Gertrud Bäumer, Adelheid, Mutter der Königreiche, Tübingen, Rainer Wunderlich Verlag, 1936 (640 S.).

4. Entsprechend der Definition von Mythos als zur Legende gewordene Begebenheit oder Person von weltgeschichtlicher Bedeutung (s. Wahrig).

5. Angelika Schaser, Frauenbewegung in Deutschland, 1848-1933. Darmstadt, Wissenschaftliche Buchgesellschaft, 2006, S. 83.

6. „Bäumer schließt sich der ,Freisinnigen Vereinigung’ an, mit der Naumann 1903 seinen ,Nationalsozialen Verein' verschmolzen hat, und lässt sich 1909 in den Vorstand der Gesamtpartei wählen. 1912 bittet Naumann sie um ihre Mitarbeit am kulturellen Teil der von ihm herausgegebenen Zeitschrift Die Hilfe, die Bäumer nach Naumanns Tod 1919 zusammen mit Theodor Heuss noch bis 
Presselandschaft des Kaiserreichs als unumgängliche Persönlichkeit. Nach dem Ersten Weltkrieg wurde sie zur „Vernunftrepublikanerin“. 1919/1920 war sie Mitglied der Verfassunggebenden Nationalversammlung, bis 1932 gehörte sie dem Reichstag an (als Mitbegründerin der Deutschen Demokratischen Partei, später deutsche Staatspartei) und war 1920 bis 1933 Ministerialrätin im Reichsinnenministerium (die höchste staatliche Position die eine Frau damals innehaben konnte, sie war für die Referate Jugendwohlfahrt und Schulwesen zuständig). Daneben war sie von 1926 bis 1933 Delegierte der Reichsregierung beim Völkerbund in Genf. Am 21. April 1933 wurde Gertrud Bäumer aufgrund des Gesetzes zur Wiederherstellung des Berufsbeamtentums aus ihrer Stellung im Reichsministerium des Inneren entlassen. Dies löste damals heftige Reaktionen aus, ja Proteste, die alle Bäumer als prominentes Opfer des NS darstellen. Nach dem Krieg jedoch wurde sie des Mitläufertums bezichtigt: eine Kontroverse bezüglich ihrer ununterbrochenen publizistischen Tätigkeit während des 3. Reichs entbrannte. Als sie 1933 entlassen wurde, konnte sie sich den gewohnten Lebensstil in Berlin nicht mehr leisten und zog mit ihrer Freundin Gertrud Hammer von Sanden nach Schlesien. 1937 ließ sie die Reichsschrifttumskammer wissen, dass sie nun hauptberuflich Schriftstellerin sei und dass sie mit dieser Tätigkeit den größten Teil ihrer Einnahmen erziele. Sie war tatsächlich eine äußerst erfolgreiche Autorin. Ihre Publikationen erzielten hohe Auflagen. Jahr für Jahr schrieb Gertrud Bäumer mindestens ein Buch - für ihre Arbeit standen ihr wechselnde Sekretärinnen zur Verfügung. Im Herbst 1941 hatte diese Stelle eine junge Frau jüdischer Abstammung inne (Isa Joschkowitz). Bäumer gehörte zwar zur Reichsschrifttumskammer, hatte dort aber viele Feinde. Die Kammer veranlasste das Propagandaministerium und die geheime Staatspolizei, Auskünfte über „eine der wildesten Demokratinnen“ einzuholen. Die ehemalige Vernunftrepublikanerin (das war sie schon eher) wandte sich immer mehr dem Mystischen, dem Irrationalen zu - die Bedeutung und das Wirken der Frau in der Entfaltung des Christentums und in der Reichsentwicklung standen in ihren historischen Arbeiten im Mittelpunkt.

Während der Jahre 1933-1945 entstanden in Deutschland eine Vielzahl von historischen Romanen und Abhandlungen zum Mittelalter. Schriftsteller aller Couleur griffen auf historische Stoffe zurück. Gertrud Bäumer bildete mit ihrer Themenwahl keine Ausnahme, sondern lag im Trend. ${ }^{7}$ Sie wählte mit der Adelheid den Beginn der deutschen Geschichte mit dem Zerfall des karolingischen und der Konsolidierung des ostfränkischen-deutschen Reiches. In einer gegenwärtigen Zeit wo die Frauen aus der öffentlichen Sphäre, insbesondere der Politik, zurückgedrängt wurden, verwies die Frauenrechtlerin Bäumer auf eine mächtige Herrscherin aus der Ottonen-Zeit. Die zeitliche Distanzierung um über zehn Jahrhunderte bewirkt

1936 weiterführt", Margit Göttert, Macht und Eros. Frauenbeziehungen weibliche Kultur um 1900 - eine neue Perspektive auf Helene Lange und Gertrud Bäumer, Königstein/ Taunus, Ulrike Helmer, S. 32 .

7. Für die Schriftsteller, die man der, ,inneren Emigration“ zurechnet (oder die, die diese Bezeichnung für sich in Anspruch nehmen), ist der historische Roman das bevorzugte Genre, siehe Frank Westenfelder, Genese, Problematik und Wirkung nationalsozialistischer Literatur am Beispiel des historischen Romans zwischen 1890 und 1945, Frankfurt a. M., Peter Lang, 1989, S. 267. 
eine Art Verfremdungseffekt: es wird zwar keine direkte Kritik an der Gegenwart, aber doch indirekte Kritik geäußert. Bäumer war davon überzeugt, dass sich die Frauenbewegung aufgrund ihres organischen Charakters (Wachstums) in jeder politischen Staatsform durchsetzen würde, und sah sich selber als geistige Vertreterin des Erbes der Frauenbewegung. Sie wollte ihre Werke als Kritik am frauenfeindlichen Dritten Reich verstanden wissen - denn offener Widerstand hätte sie der verbliebenen Einflussmöglichkeiten beraubt. Wohlgemerkt kann man die Adelheid, wie alle ihre Bücher, als bildungsbürgerliche Unterhaltungsliteratur, als revisionistische, nationalistische aber auch christliche Literatur charakterisieren, als regimekritisch oder regimefreundlich interpretieren. ${ }^{8}$ Das Janusköpfige des politischen Mythos (d.h. rechte und linke, kontinuitätswahrende und emanzipatorische Elemente verbindend ${ }^{9}$ ) trägt durchaus dazu bei. Mit ihrer schriftstellerischen Tätigkeit setzte Bäumer ihren Kampf mit eingeschränkten Mitteln fort, „während eine politische Mehrheit der Frauen die erstrebten und errungenen Ziele im Stich gelassen hat", wie sie im Dezember 1943 feststellte. ${ }^{10}$ Die Verlagerung der Gegenwartsproblematik in das Mittelalter und das Herausstellen der Bedeutung der christlichen Kultur galten Gertrud Bäumer als gelungene Camouflage-Methoden. ${ }^{11}$ Die These dieses Beitrags ist daher, dass die Autorin auf verschlüsselte Weise, durch die Reaktivierung eines weiblichen politischen Mythos, versucht, gegen die Zurückdrängung der Frauen aus der öffentlichen Sphäre anzugehen. Dabei spielen sowohl Produktion als Rezeption eine Rolle (siehe die eingangs gestellten Fragen).

Kommen wir zunächst auf die Wahl des Adelheidstoffes und die damit verbundenen Schwierigkeiten zurück. Der Forschungsstand zu Kaiserin Adelheid war damals (und bleibt heute noch immer) trotz der Bekanntheit ihrer Person ziemlich spärlich. ${ }^{12}$ Gertrud Bäumer hatte also keine moderne Literatur, auch keine Historiographie zur Verfügung, als sie sich an die Arbeit machte. Die beiden Hauptquellen bildeten die so genannten Gesta Ottonis, die Taten Ottos; diese wurden 965/968 von der Nonne Hroswith von Gandersheim verfasst. Besonders wichtig ist der Nachruf Abt Odilos von Cluny, der in der ersten Jahreshälfte 1002 entstand, anscheinend auf Auftrag Adelheids persönlich (die also selber für die Legendenbildung um ihre Person sorgte). ${ }^{13}$ Gertrud Bäumer scheint insbesondere von diesem Epitaphium stark beeinflusst worden zu sein. Darüber hinaus war die

8. Angelika Schaser, Helene Lange und Gertrud Bäumer. Eine politische Lebensgemeinschaft, Köln/ Weimar/ Wien, Böhlau, 2000, S. 311-312.

9. Yves Bizeul (Hrsg.), Politische Mythen und Rituale in Deutschland, Frankreich und Polen, Berlin, Duncker \& Humblot, 2000, S. 13 und 32.

10. Schaser, Helene Lange und Gertrud Bäumer, S. 324-25. Herv. C. S.-F.

11. Schaser, „GertrudBäumer-eine der, wildesten Demokratinnen“oder verhinderte Nationalsozialistin?“ in Kirsten Helnsohn, Barbara Vogel, Ulrike Weckel (Hrsg), Zwischen Karriere und Verfolgung. Handlungsräume von Frauen im nationalsozialistischen Deutschland, Frankfurt a. M., Campus, 1997, S. 30.

12. Laut Aussage des Mediävisten Prof. Stefan Weinfurter 1999 anlässlich des 1000. Todestages von Kaiserin Adelheid. Siehe Stefan Weinfurter, „Kaiserin Adelheid und das ottonische Kaisertum“, in Hagen Keller, Christel Meier (Hrsg.), Frühmittelalterliche Studien. Bd.33. Berlin, Walter de Gruyter, 1999, S. 1-19. Literatur über Adelheid gebe es grundsätzlich erst nach 1950.

13. Odilo von Cluny, Heiliger, Epitaphium Domine Adelheide Auguste (Die Lebensbeschreibung der Kaiserin Adelheid / Odilo Abt von Cluny. Bearb. von Herbert Paulhart, Graz / Köln, Böhlau, 1962). 
beschränkte Quellenlage nicht unbedingt ungünstig ${ }^{14}$ : Hier kann man verweisen auf die Definition des politischen Mythos als emotional aufgeladene Narration, die die historische Wirklichkeit nicht den Tatsachen gemäß, sondern in einer selektiven und stereotypisierten Weise interpretiert und dieser „mythischen Lesart der Wirklichkeit“" einen Anschein von Historizität verleiht. ${ }^{15}$ Die Quellen jedenfalls preisen Adelheid als Idealtypus einer Kaiserin. Gott selbst habe sie für dieses Amt ausersehen, sie nach verschiedenen harten Prüfungen immer wieder erhöht und ihr die Spitze des Kaiserreiches anvertraut, die ihr zugefügten Erniedrigungen habe sie nur deshalb zu erdulden gehabt, um in herrlicher Weise erhöht zu werden; daher sollte man sie als die „kaiserlichste aller Kaiserinnen“ bezeichnen und ehren, fordert zum Beispiel Abt von Cluny den Leser auf. ${ }^{16}$ Genau diese Dialektik durchzieht den Adelheid Band Bäumers wie ein roter Faden. Man kann hier zugleich eine Parabel der Frauenbewegung erkennen - ähnlich der später gebrauchten Wellenmetapher vielleicht sogar die Utopie des Sieges des Feminismus über den Patriarchalismus. Jedenfalls schildert Gertrud Bäumer, wie die früheren Biografen, Adelheid in aktiver Kaiserinnen Politik. Indem sie auf die integrative Funktion des politischen Mythos baut, trägt sie zum geistigen Zusammenhalt der Frauenbewegung im Dritten Reich bei.

Tatsächlich fungiert Adelheid als Vorbild für die ,alte“ Frauenbewegung: Bäumer benutzt Codes, die alle verstehen können, wie den der „geistigen Mütterlichkeit“" (von dem noch die Rede sein wird). Sie lobt traditionelle, christliche Werte. Und wie könnte man sich den Erfolg des Werks anders erklären, als mit der Identifikation eines großen Teils der Leserschaft? Der Figurenmythos Adelheid bietet sich auch aufgrund ihres abenteuerlichen Lebens besonders gut für Bäumers Vorhaben an. Sie erlebt, wagt und bewirkt viel, während die Frauenbewegung ihrer Aktionsmöglichkeiten beraubt ist und auch viele Leserinnen (und Leser) unter dem Nationalsozialismus in einer Art Starre verharren müssen.

Das 640 Seiten starke Werk Adelheid, Mutter der Königreiche zerfällt in sechs Abschnitte. In den zwei ersten Abschnitten (von Seite 1 bis 183) steht noch Adelheids Mutter, Königin Bertha im Mittelpunkt. Ab dem dritten Abschnitt wird Adelheid selbst zur Protagonistin. Gertrud Bäumer spinnt die Genealogie in den nächsten Abschnitten weiter, in dem sie dann den Sohn Adelheids, Otto II., schließlich ihren

14. Das spärliche Fließen der Quellen musste übrigens keine ungünstige Vorbedingung sein, da die Fantasie der Autorin die Lücken füllen konnte, siehe Heidi Beutin, „Ein Mittelalter für die Frauenbewegung?“, in Heidi Beutin, „Als eine Frau lesen lernte, trat die Frauenfrage in die Welt". Beiträge zum Verhältnis von Feminismus und Literatur anhand von Schriften Marie von Ebner-Eschenbachs, Lily Brauns, Gertrud Bäumers, Gerhard Anton von Halems, Christoph Martin Wielands und Jutta Heckers, Hamburg, von Bocken, 1990, S. 78.

15. Heidi Hein-Kirchner, Politische Mythen. Bundeszentrale für politische Bildung. URL: [http://www. bpb.de/apuz/30604/politische-mythen]. Ohne Verlagsangabe (o.V.), ohne Seitenangabe (o.S.): 2. März 2007 (vgl. mit Heidi Hein-Kirchner, „Überlegungen zu einer Typologisierung von politischen Mythen aus historiographischer Sicht - ein Versuch“, in Heidi Hein-Kirchner, Hans Henning Hahn (Hrsg.), Politische Mythen im 19. und 20. Jahrhundert, Marburg, Herder Institut, 2006, S. 407-424.

16. Weinfurter, „Kaiserin Adelheid“, S. 2. 
Enkelsohn Kaiser Otto III., mit einbezieht. ${ }^{17}$ Der Roman ist zugleich dicht und langatmig: obwohl alles auf ihre Krönung hinaus läuft, werden Otto und Adelheid erst auf Seite 472 Kaiser und Kaiserin (!) Dies konnte allerdings auch den Zensor entmutigen: am Anfang des Romans stirbt Adelheids Vater Rudolf ... 16 Seiten lang währt seine in Weihrauch und Gesänge gehüllte Agonie.

Trotzdem kann man schon aus den beiden ersten Abschnitten herauslesen, dass Adelheid ein abenteuerliches Leben führte, welches sich in die Historie einschreibt. Schon der Tod des Vaters brachte einschneidende Veränderungen mit sich, denn König Hugo von Italien sah nun seine Chance gekommen, ein italo-burgundisches Großreich zu bilden. Er zwang die verwitwete Mutter Adelheids, Bertha, zur Heirat (mit sich selbst). Seinen etwa zehnjährigen Sohn Lothar verlobte er mit der kleinen Adelheid, die damals sechs oder sieben war. Von diesem Zeitpunkt an wurde sie am Hof in Pavia erzogen und ausgebildet. Adelheids Brüder, Konrad und Rudolf, konnten entkommen und flüchteten sich an den Hof Ottos von Sachsen. Adelheid wurde in Pavia allerdings größte Aufmerksamkeit und Fürsorge entgegengebracht. Am italienischen Königshof wuchs sie wie eine Prinzessin heran, erhielt die bestmögliche Ausbildung; gerade diese Nähe zur imperialen Tradition in Pavia scheint sehr beachtenswert. Adelheid erlebte nämlich mit, wie auch Hugo, ihr Schwiegervater, daran anknüpfte und mehrmals versuchte, die Kaiserkrone in Rom zu gewinnen. Ihr Lebenskreis war von imperialem Rangdenken erfüllt. ${ }^{18}$ Anlässlich ihrer Verlobung 937 wurde Adelheid mit umfangreichen, königlichen Besitzungen in Oberitalien ausgestattet. Diese Besitzungen, wie ihre späteren Rechtshandlungen zeigen, wurden ihr zur uneingeschränkten Verfügung übertragen. Sie allein und persönlich konnte darüber bestimmen, daher galt sie als die reichste Frau des Abendlandes. Von Kindesbeinen an verfügte sie über ein eigenes Königreich, und von Anfang an wuchs sie in die Rolle der Königin von Italien hinein. 947, Adelheid war damals gerade 16 Jahre, ihr Gemahl etwa 20 Jahre alt, wurde die Hochzeit vollzogen. Etwa zur selben Zeit starb Hugo (der Vater ihres Gatten) und Lothar übernahm die alleinige Herrschaftsgewalt. Adelheid rückte damit in die Rolle der regierenden Königin von Italien, und zwar im Sinne einer Mitherrscherin: daher betitelt Gertrud Bäumer den dritten Abschnitt „Consors regni“. ${ }^{19}$ Der Roman beschreibt eine glückliche Ehe. Doch im Jahr 950 wurde Adelheids Mann, nach nur drei Ehejahren, vergiftet. Markgraf Berengar von Ivrea wollte seinen Sohn Adalbert mit der jungen Witwe verheiraten, um über sie die italienische Krone zu

17. Erster Abschnitt: Königin Bertha. Zweiter Abschnitt: die Königin der Lombardei. Dritter Abschnitt: Consors regni. Vierter Abschnitt: Otto der Große. Fünfter Abschnitt: die Kaiserin. Sechster Abschnitt: Mutter der Königreiche.

18. Weinfurter, „Kaiserin Adelheid“, S. 6.

19. Bäumer, Adelheid, S. 183 ff. 
erlangen. ${ }^{20}$ Adelheid lehnte jedoch ab, worauf Berengar sie auf seiner Burg in Garda gefangensetzte. ${ }^{21}$

Spätestens hier beginnt die Legendenbildung: Königin Adelheid wird es nämlich gelingen, aus der Gefangenschaft zu flüchten. Politische Mythen beziehen sich auf meisterhafte, heldenhafte Taten, die auch über den Tod des jeweiligen Helden hinaus Geltung und Gültigkeit tragen und Einfluss auf die gegenwärtige Gesellschaft haben; auch wird diese Episode im Roman ausführlich beschrieben. Die Autorin schildert im Detail, wie zwei Männer im Hof ihr durch das Anordnen eines Holzhaufens Botschaften senden (Adelheid beobachtet sie von ihrem Turmfenster aus), um ihr zu verstehen zu geben, dass man von ihr erwarte, dass sie einen Ausweg finde und sich bis Mantua zu Fuß durchschlüge. Die letzte Botschaft lautet „der Fels hat Höhlen: grabt!"22 Man lässt Adelheid und ihren Mitgefangenen (dem Priester Martin und der Dienerin Bonella) auf dem Boden des Eimers in dem der Unrat aus dem Kerker hinausbefördert wird, ein Eisen zukommen und sie graben hinter dem Altar ihrer Zelle, wo sie ihren Versuch immer wieder verbergen können... Doch geht die Arbeit nur langsam voran. Verzweiflung und Not bemächtigen sich der Königin, also wühlt sie in Schmutz und Gestank des Kerkers mit verschlissenen Kleidern, verwildertem Haar, zerrissenen Händen wie ein Tier. Doch weicht die realistische Beschreibung bald einer mystischen und Adelheids Visionen rücken in den Mittelpunkt: ,. [...] in diesem Licht stand die Gestalt des Kreuztragenden vor ihr: er war der Allerverachtetste und Unwerteste. Dann konnte sie wohl eine Würde über alle Würde fühlen um ihrer Not willen, die über alle Not war." ${ }^{23}$ Dank ihres tiefen Glaubens und unbeugsamen Willens konnte Adelheid schließlich aus Garda entkommen und auf die Burg Canossa flüchten. Von Canossa aus rief sie König Otto I., der ihren Bruder Konrad erzogen hatte, zu Hilfe. Man vermutet, dass sie ihm eine Verbindung in Aussicht stellte, was mit anderen Worten bedeutet, dass sie sich ihren neuen Gatten selbst wählte (!). Otto besiegte Berengar, heiratete Adelheid 951 in der lombardischen Königsstadt Pavia und übernahm die langobardisch-italienische Königswürde (setzte aber Berengar als seinen Stellvertreter ein). Mit dieser Heirat wurde die Grundlage für die Verbindung des ostfränkischen und des lombardischen Königreiches, dem Kernstück des späteren Imperium Romanum des Mittelalters gelegt. Adelheid erscheint als diejenige, die es Otto überhaupt ermöglichte, die Kaiserkrone aufzusetzen; in der Forschung wird hingegen ziemlich einhellig die Meinung vertreten, Ottos Italienpolitik sei im Kern ganz unabhängig von Adelheid gewesen. Doch eröffnete Adelheid dem sächsischen Königtum ungeahnte neue Dimensionen und Perspektiven, da sie der Schlüssel war, der das Tor zur romanischen Welt öffnete als Königin von Italien. ${ }^{24}$ Aufgrund

20. Bemerkenswerter Weise steht Berengar unter der Fuchtel seiner Frau Willa, die im Roman als Gegenfigur zu Adelheid fungiert (während die Historiographie eher Kaiserin Theophanu als solche betrachtet). Willa beeinflusst Berengar, ja sie manipuliert ihn sogar, s. Gertrud Bäumer, Adelheid, S. 174. Sie hegt einen tödlichen Hass auf die jüngere Adelheid; Berengar und Willa streben nach der Kaiserwürde, ibid., S. 131, 137, 139.

21. Ibid., S. $185 \mathrm{ff}$.

22. Bäumer, Adelheid, S. 190.

23. Ibid., S. 191.

24. Weinfurter, „Kaiserin Adelheid“, S. 8-9. 
ihrer gelungenen Flucht wird Adelheid eine Heldin für das Volk und bringt diese Macht als Mitgift in die Ehe mit Otto mit. ${ }^{25}$ Der starke, feste Glaube Adelheids an das Kaisertum durchzieht den Band wie ein roter Faden. Sie erscheint an vielen Stellen als Erbin, da sie von Vater und Mutter dazu erzogen wurde, ein Reich der Christenheit und einen Kaiser zu wollen. ${ }^{26}$ Sie glaubt also von Kind an an das Kaisertum ${ }^{27}$; Kaisertum, das das Reich der Christenheit neu begründen wird, das Reich „,von Mitternacht zu Sonnenaufgang“, als „,das Bollwerk gegen die Heiden“. ${ }^{28}$ Was die zeitgenössische Rezeption des Romans zur Zeit seiner Erscheinung betrifft, liegt es nahe, Adelheids Flucht aus der Gefangenschaft als Aufruf zum Handeln aufzufassen, als Botschaft die besagt „Helfe dir selber“? Wie Bäumer Spezialistin Angelika Schaser feststellt: „Das herausragende Kennzeichen ihrer historischen Schriften, die durchaus wissenschaftliche Ambitionen haben, ist die Hervorhebung der mittelalterlichen Frauenfiguren als handelnde Personen. “29

Adelheid und Otto hatten vier gemeinsame Kinder: Heinrich (952-954), Bruno (953-953), Mathilde (spätere Äbtissin von Quedlinburg, 954-999), Otto II. (später Kaiser des Heiligen Römischen Reiches, 955-983, der in Abschnitt fünf und sechs des Romans eine wichtige Rolle spielt). Adelheid ist also leibliche Mutter, noch bevor sie zur „Mutter der Königreiche“ wird (siehe den Titel des Romans). Die Mütterlichkeit ist ein wichtiges Thema des Werks, beinhaltet Schmerz und Glück zugleich, Adelheid benennt es "Mutterschicksal“". ${ }^{30}$ Der Verlust eines Kindes wird von der Mutter ganz anders empfunden als von dem Vater: als Bruno, der zweite Sohn Adelheids und Ottos, im Kinderbett stirbt, trauert Adelheid, wobei Otto ihren Schmerz nur schwer verstehen kann. ${ }^{31}$ Auch spürt sie, kann es diesbezüglich keine Verständigung zwischen den Eheleuten geben; eine Grenze ist gesetzt. Dies mag einerseits vereinfachend erscheinen; andererseits geht die Autorin manchmal auch gegen Stereotype an. Weint Adelheid trotz aller Stärke mehrfach ${ }^{32}$, so tut Otto das überraschenderweise auch. ${ }^{33}$ Oft erscheint die schmale Kaiserin Adelheid kräftiger als Otto der Große; seine düstere Schwere reizt sie, sie ist trotz körperlicher Unterlegenheit kräftiger als er. ${ }^{34}$ Auch Adelheids Mutter Bertha ist, wie schon erwähnt, eine wichtige Figur, die ihre Tochter prägt. ${ }^{35}$ Die Mütterlichkeit insbesondere begründet den Unterschied zwischen Frau und Mann, liest man im Roman. ${ }^{36}$ Die alte Frauenbewegung baute auf diesen Differenzialismus: Anhänger des ,differenzialistischen Feminismus“ gingen von einer grundsätzlichen Verschiedenheit der Geschlechter aus und postulierten somit die Gleichwertigkeit,

25. Bäumer, Adelheid, S. 281.

26. Ibid., S. 120, S. 132.

27. Ibid., S. 219, S. 223

28. Ibid., S. 212-213.

29. Schaser, Helene Lange und Gertrud Bäumer, S. 311.

30. Bäumer, Adelheid, S. 321.

31. Ibid., S. 420

32. Ibid., S. 500, S. 515.

33. Ibid., S. 534.

34. Ibid., S. 345.

35. Ibid., S. 433.

36. Ibid., S. 436. 
nicht aber Gleichberechtigung. Gertrud Bäumers Lebensgefährtin Helene Lange hatte um die Jahrhundertwende das Konzept der ,geistigen Mütterlichkeit“ geprägt. Um ihren Anspruch auf weibliche Mitgestaltung der Gesellschaft zu untermauern, berief sie sich auf eine Mütterlichkeit, die nicht mehr an die biologische Mutterschaft gebunden war, sondern angeborenes weibliches Verhalten mit erworbenem Wissen verband. ${ }^{37}$ Geistige Mütterlichkeit konnte sich nicht nur innerhalb der Familie entfalten, sondern auch in jedweder Form sozialer und pädagogischer Arbeit und Berufstätigkeit. Helene Lange sah in der Natur der Frau Kräfte, die das Potential eines höchsten Korrektivs der gesellschaftlichen Fehlentwicklungen innehatten. Aber um diese Energie einsetzen zu können, bedürfe die Frau ganz anderer Machtmittel, einer ganz anderen Stellung im Staat, als sie heute habe. Ihr Fazit lautete daher deutlich:

Den Weg dazu hat sie eingeschlagen, als sie in den vierziger Jahren des vorigen Jahrhunderts zuerst Hand an die Politik legte. Die Aufgabe unseres Jahrhunderts wird es sein, diesen Weg zum Ziel zu führen. Und darum muss die Parole für die Frauen auch fernerhin lauten: ,Hand an die Politik.' 38

Sie intendierte Revolution, allerdings im Gewand der Reform, welches sie gesellschaftsfähig und effizient machte. Die von Helene Lange vorgegebene Strategie, zwar zielstrebig und konsequent, aber langsam und bedacht vorzugehen, erwies sich langfristig als erfolgreich. Die deutschen Frauen erhielten das aktive und passive Wahlrecht in der tief erschütterten demokratisierten Nachkriegsgesellschaft; allerdings setzten die nationalsozialistischen Machthaber spätestens ab 1933 alles daran, sie aus der Öffentlichkeit zurückzudrängen.

Oft werden historische Protagonistinnen während politischer Umbrüche zu mythischen Heldinnen. Als symbolträchtige Sujets aufgegriffen von Kunst, Musik und Literatur, erwecken sie ein breiteres öffentliches Interesse. Derartige Frauengestalten dienen gewissermaßen als Deutungsmodelle oder Indikatoren unterschiedlicher Phänomene einer gerade aktuellen Gegenwart. Die Themenkomplexe „Frauen und Politik“ oder „Frauen und Macht“ spielen eine wichtige Rolle im Adelheid Band Gertrud Bäumers; insbesondere die Regentschaft der Kaiserinnen (983-994). Nach dem frühen Tod Ottos II. übernahmen Adelheid und ihre Schwiegertochter Theophanu die Regentschaft für den noch minderjährigen Otto III. Auf diese Art und Weise wollten sie die Macht und die Krone des Reiches für die Dynastie der Ottonen erhalten. Gemeinsam mit dem Erzbischof Willigis von Mainz führten sie die Regierungsgeschäfte für den minderjährigen Kaiser, bis Adelheid sich nach Italien zurückzog. ${ }^{39}$ Dort wurde sie Statthalterin von Italien. 991 kehrte sie an den Kaiserhof zurück, als ihre Schwiegertochter Theophanu 35-jährig starb. Bis zur Volljährigkeit ihres Enkels Otto III. führte die (alte) Kaiserin die Regierungsgeschäfte. Konkurrenz

37. Michaela Karl, Die Geschichte der Frauenbewegung, Stuttgart, Reclam, 2011, S. 83.

38. Die Frau, Monatsschrift für das gesamte Frauenleben unserer Zeit, 1904, S. 358.

39. Im Harz entstand nach 983 eine umfangreiche Münzprägung, die neben dem Namen des noch jungen Otto III. auf der anderen Seite den seiner Großmutter Adelheid lesen lässt, die sogenannten OttoAdelheid-Pfennige. Auf welcher Rechtsgrundlage diese entstanden, ist noch nicht endgültig geklärt, doch hatte die Prägung auf der Grundlage der Silbervorkommen im Harz einen sehr großen Umfang und wurde an vielen Orten nachgeahmt. 
und Zusammenarbeit zwischen beiden Frauen werden im Roman beschrieben. Schon im zehnten Jahrhundert setzte Abt Odilo dieses Spannungsverhältnis als stilistisches Mittel ein, wobei ihm Theophanu als Meisterin über Machenschaften, machthungrige Intrigantin usw. erscheint. ${ }^{40}$ Sie habe ihrer Schwiegermutter das ganze Witwengut wegnehmen wollen (wahrscheinlicher ist, dass Theophanu Adelheid daran hindern wollte, den Stiftungen noch mehr Schenkungen zu machen - Adelheid ihrerseits vertrat die Meinung, so mache man sich Freunde und Verbündete). In der Forschung wurde diese Auseinandersetzung beschrieben, ja sogar mit dem berühmten Zwist zwischen Kriemhild und Brunhilde in Verbindung gesetzt. In den letzten Jahren gilt die Sympathie der Historiker eindeutig Theophanu, in der man ein Element politischer und kultureller Modernität sieht. ${ }^{41}$ Bei Gertrud Bäumer jedoch spielt Adelheid die Hauptrolle. Theophanu, die Griechin, wird dennoch nicht kritisiert: der Roman geht auf die Spannung zwischen Konkurrenz und Versöhnung ein und zeigt, dass Adelheid persönliche Interessen hintanstellen konnte, um die unitas imperii zu sichern. ${ }^{42}$ Man konstatiert hiermit eine bemerkenswerte Verschiebung in der Bewertung Adelheids, die in ihrer eigenen Zeit außerordentliche Hochschätzung genoss. Ihr wurde uneingeschränkte Verehrung entgegengebracht und am Ende sogar ein Rang noch vor dem Kaiser selbst eingeräumt. Dieses ist auch das Ziel Gertrud Bäumers. Heute werden eher Vorbehalte gegenüber ihrer - im Vergleich zu Theophanu - engen Welt und politischen Rückwärtsgewandtheit geäußert. ${ }^{43}$ Die Forschung hat den Adelheid-Mythos also zum Teil dekonstruiert. Doch leistet Bäumer 1936 gerade umgekehrte Arbeit, indem sie ihn rekonstruiert. Sie setzt auf Klugheit und Bildung der weiblichen Figuren, ein weiterer Schwerpunkt der ,alten“ Frauenbewegung, die als „Bildungsbewegung“ galt. ${ }^{44}$ Adelheid selber hat von Kind auf Latein gelernt, sie liest viel und setzt im Roman ihre Schreiben selber auf (in Wirklichkeit dürfte sie ihre Anweisungen Notaren und Kapellänen diktiert haben). ${ }^{45}$ Doch sie ist nicht die einzige kluge und gebildete Frauenfigur des Romans. Insbesondere die Äbtissinnen und Nonnen sind es auch: „Nie hatte sie an einer Stelle ein so lebendiges und beglücktes geistiges Leben gespürt wie es unter der jungen klugen und hochgebildeten Äbtissin die Nonnen von Gandersheim beseelte." 46 Dies schreibt sich durchaus in den Kontext des Geisteslebens im Ottonenreich ein: große Klöster im Westen und Süden übernahmen damals die karolingische Bildungstradition und hielten dieses Erbe aufrecht, ja aktivieren es, so dass es zu einem regelrechten Bildungsaufschwung

40. Die in der früheren Literatur oftmals geschilderte Feindschaft zwischen den beiden Frauen kann nicht belegt werden und wird nur von Odilo von Cluny beschrieben. Adelheid führte weiterhin die Regierungsgeschäfte in Italien und hielt zum Teil sogar Hoftag mit Theophanu.

41. Weinfurter, „Kaiserin Adelheid“, S. 10.

42. Bäumer, Adelheid, S. 578.

43. Stellt sich tatsächlich die Frage, was Adelheid für das Kaisertum und die Ottonen Dynastie bedeutete, scheint die Frage nach den Kategorien Vergangenheit und Zukunft weniger relevant. Weinfurter, „Kaiserin Adelheid“, S. 4.

44. Schaser, Frauenbewegung in Deutschland, S. $23 \mathrm{ff}$.

45. Bäumer, Adelheid, S. 142. Weinfurter, „Kaiserin Adelheid“, S. 2.

46. Bäumer, Adelheid, S. 513. 
kam. ${ }^{47}$ Gerade auch durch ihre Intelligenz sind die Romanfiguren (Beatrix, Bathildis, Hadwig, Judith, Gerberga... ${ }^{48}$ ) die besseren Politikerinnen - zumindest, das deutet Gertrud Bäumer durch die weiblichen Beispiele im Adelheid-Roman an, täten die männlichen Herrscher gut daran, öfter ihre Ratschläge einzuholen und zu befolgen. Man sieht hier wiederum, warum politische Mythen gerade in Krisenzeiten und gesellschaftlichen und politischen Umbruchsphasen häufig Konjunktur erleben. Sie wirken orientierungsgebend für soziale Gruppen. ${ }^{49}$ Gertrud Bäumer konnte wohl kaum erwarten, dass die nationalsozialistischen Machthaber sich ihre mehr oder weniger verschlüsselten Botschaften zu Herzen nähmen, doch konnte sie ihrem Publikum durchaus zu verstehen geben, was sie von der Zurückdrängung der Frauen aus der öffentlichen Sphäre hielt. Ihre Meinung als bekannte Frauenrechtlerin und Publizistin zählte durchaus; auch war ihre Breitenwirkung im Vergleich zur universitären Mediävistik eine viel größere, weil sie sich die Freiheit nahm, die Lücken der Überlieferung durch Dichtung zu überbrücken. ${ }^{50}$

Entscheidend für die Stoffwahl dürfte also gewesen sein, dass zu keiner Zeit im Mittelalter Frauen des Könighauses eine derart hervorragende Stellung in der Politik eingenommen haben wie in der Ottonenzeit. Entsprechend ließ Bäumer in ihrem Werk das hellste Licht auf diese historischen Persönlichkeiten fallen, an erster Stelle auf die Titelgestalt Adelheid, ferner auf ihre Schwiegertochter, Kaiserin Theophanu, sowie auf andere Königinnen, Herzoginnen, Äbtissinnen usw. ${ }^{51}$ Kurz bevor sie sich des Adelheid-Mythos annahm, hatte Bäumer Paarbiographien aus dem frühen Mittelalter veröffentlicht (z.B. Heinrich II und Kunigunde), in denen sie sowohl versuchte, das gemeinsame Wirken dieser Paare zu verdeutlichen, wie die ungleiche Quellenlage auszugleichen. ${ }^{52}$ Durch die Hervorhebung der Frauengestalten wollte Bäumer „das deutsche Volk“ darüber aufklären, „wie sich in der Mitregierung, die Sitte und Recht der Königin ausdrücklich übertrug, ein tiefer und sicherer Sinn für die wechselseitige Ergänzung der Geschlechter in der Gestalt des Volksschicksals ausprägte. “" ${ }^{33}$ In der Adelheid versuchte Bäumer ihr Frauenideal zum vollkommenen Ausdruck zu bringen, mit folgenden Grundzügen: der Teilhabe an der politischen Macht, der Mütterlichkeit (Adelheid ist leibliche Mutter, sie übernimmt die Pflege der Kinder anderer Mütter ${ }^{54}$, wird zur „Mutter der Königreiche“), der Pflege von Verwundeten und Sorge für Kranke und Arme. ${ }^{55}$ Elemente ihres Frauenbildes teilte die Autorin auch anderen Frauen zu (z.B. der Nonne Hrotsvith als Schöpferin

47. Josef Fleckenstein, Horst Fuhrmann, Joachim Leuschner, Deutsche Geschichte, Bd 1, Mittelalter, Göttingen, Vandenhoeck \& Ruprecht, 1985, S. 144.

48. Bäumer, Adelheid, S. 570 (Beatrix), S. 553 (Bathildis), S. 545 (Hadwig), S. 535 (Judith), S. 505 (Gerberga) ...

49. Und sind daher ein zentrales Mittel zur Massenkommunikation, Heidi Hein Kirchner, Politische Mythen.

50. Schaser, Helene Lange und Gertrud Bäumer, S. 311.

51. Beutin, Als eine Frau lesen lernte, S. 81.

52. Bäumer, Männer und Frauen im geistigen Werden des deutschen Volkes, Tübingen, 1934.

53. Ibid., Vorwort, zitiert in Angelika Schaser Helene Lange und Gertrud Bäumer, S. 309.

54. Gertrud Bäumer, Adelheid, S. 422 f.

55. Ibid, S. 496. 
dichterischer Werke ${ }^{56}$ ) sowie der mythologischen Gestalt der Gottesmutter. ${ }^{57}$ Um das Bild noch deutlicher zu gestalten, entwarf sie Gegenbilder in Gestalt einiger nicht autonom über sich selbst verfügender Frauen (Ottos Tochter Liutgart z.B.). Dies erscheint im Nachhinein recht konventionell und wenig gewagt: Rita Thalmann, durch ihre Arbeiten über Jochen Klepper und Gustav Frenssen Kennerin der Literatur dieser Zeit, urteilte daher, dass Bäumer „brave Bücher über Frauen und ihre Geschichte" schriebe. ${ }^{58}$ Doch kann man mit dieser Aussage den Erfolg des Adelheid Romans kaum erklären: bis 1945 erschienen, wie schon erwähnt, sechs Auflagen (1942 wurde eine Sonderauflage für die Wehrmacht hergestellt), 1941 diente sie als Vorlage für ein Opernlibretto. Nach dem Krieg wurde es weiter verlegt, 1955 erreichte die Auflage eine Höhe von 100000 Exemplaren. Adelheid erschien insofern als unangreifbarer Mythos, als sie schon zu Lebzeiten wie eine Heilige verehrt wurde; sie wurde im Hinblick auf das Kaisertum als ein Werkzeug Gottes angesehen. ${ }^{59}$ Gertrud Bäumer schildert ihre Frömmigkeit und zeigt, welche Verehrung ihr entgegengebracht wird; ihr eigener fester Glaube durchzieht das Buch wie ein roter Faden. Von Kind an hegt Adelheid das Ideal des Kaisertums, das das Reich der Christen neu begründen wird. Und sie trägt zur Realisierung bei. Percy Ernst Schramm, der zu den international renommiertesten deutschen Mittelalterhistorikern im 20. Jahrhundert zählt, lobte das Buch Adelheid, und attestierte ihm nicht nur Wissenschaftlichkeit, sondern erkannte das Oppositionelle: „Dazu machen Sie deutlich“ schrieb er am 5. Dez. 1936 an Bäumer, „welche Kraft und Seligkeit das Christentum spenden kann - das ist die beste Polemik gegen die, die von solchen Dingen nichts ahnen." ${ }^{60}$ Dieses Urteil zeigt, dass der AdelheidRoman für deutungsfähige und -willige durchaus ein „anderes Deutschland“ darstellen konnte, was seinen Erfolg erklären kann. Die Frage zu Bäumer selber: „Rückzug ins Mittelalter oder Fortsetzung der Arbeit für die Frauenbewegung mit anderen Mitteln?" (Heidi Beutin) kann eben nicht eindeutig beantwortet werden, weil beide Antworten gültig sind. Im letzten Dialog, den Bäumer Adelheid mit Abt Odilo von Cluny führen lässt, betont diese rückblickend sowohl ihre Macht als ihre Ohnmacht:

„Ich war dreimal Kaiserin des Römischen Reiches: mit meinem Gemahl, für meinen Sohn, für meinen Enkel. Wir haben das Schwert geführt gegen heidnische Völker - hätten sie sonst nicht die Christenheit vernichtet? [...] Und wir haben zu Felde gelegen - christliche gegen christliche Völker. [...] Und du sahst das Schlimmste, Odilo, den Kampf des Bruders gegen den Bruder, der Söhne gegen den Vater? Wie wenig vermochten wir Frauen!“"61

56. Ibid, S. 513 .

57. Ibid, Adelheid, S. 321, vgl. mit Heidi Beutin, Als eine Frau lesen lernte, S. 81-82.

58. Rita Thalmann, Frausein im 3. Reich, München 1984, S. 96 zitiert in Angelika Schaser, Helene Lange und Gertrud Bäumer, S. 311.

59. Weinfurter, „Kaiserin Adelheid“', S. 11.

60. Schaser, Helene Lange und Gertrud Bäumer, S. 311. Für die Nazis war Jesus ein Jude, und das Christentum musste „entjudaisiert“ werden, Johann Chapoutot, La loi du sang, Paris, Gallimard, 2014, S. 152 ff. Mit ihrer Verherrlichung des herkömmlichen Christentums wendet sich Gertrud Bäumer durchaus gegen die „Deutschen Christen“ und die „Reichskirche“.

61. Bäumer, Adelheid, S. 634. 
Diese Worte Adelheids dürften die Stimmung Bäumers ziemlich genau wiedergeben, verband sich bei ihr doch die Sehnsucht nach einer einst ausgeübten politischen Macht mit der Einsicht in die Begrenztheit des eigenen Wirkens. ${ }^{62}$ Ihre politischen Freundinnen kritisierten zunehmend ihre vermeintliche Übereinstimmung mit dem NS-Regime; sie selber war überzeugt, eine schwere Bürde zu tragen, nämlich das Erbe der bürgerlichen Frauenbewegung zu bewahren - was ihr bis 1944 mit relativem Erfolg auch gelang. ${ }^{63}$

62. Schaser, Helene Lange und Gertrud Bäumer, S. 310.

63. Insbesondere durch die Herausgeberschaft der FRAU, siehe Schaser, „Gertrud Bäumer - eine der ,wildesten Demokratinnen“ oder verhinderte Nationalsozialistin?“. Siehe auch Christina StangeFayos, „Le mensuel Die Frau, Berlin 1930-1944, entre adaptation et immunité“, in Michel Grunewald, Olivier Dard, Uwe Puschner, Confrontations au national-socialisme dans l'Europe francophone et germanophone, Vol. 3, La famille libérale/ modérée/ européiste, Bern, Peter Lang, 2018, S. 225-240. 
